\title{
Glioblastoma, IDH-Mutant
}

National Cancer Institute

\section{Source}

National Cancer Institute. Glioblastoma, IDH-Mutant. NCI Thesaurus. Code C39751.

A glioblastoma arising from a lower grade astrocytoma. It is more commonly seen in younger patients and is associated with IDH1 or IDH2 gene mutations. 